\title{
Comment bâtir un internet low tech
}

How to Build a Low-tech Internet

\section{Kris De Decker}

Traducteur : Olivier Morice

\section{(2) OpenEdition}

Journals

Édition électronique

URL : https://journals.openedition.org/tc/8489

DOI : $10.4000 /$ tc. 8489

ISSN : 1952-420X

Éditeur

Éditions de l'EHESS

Édition imprimée

Date de publication : 6 juin 2017

Pagination : 216-235

ISBN : 978-2-7132-2707-3

ISSN : $0248-6016$

\section{Référence électronique}

Kris De Decker, "Comment bâtir un internet low tech », Techniques \& Culture [En ligne], 67 | 2017, mis en ligne le 06 juin 2019, consulté le 29 septembre 2022. URL : http://journals.openedition.org/tc/8489 ; DOI : https://doi.org/10.4000/tc.8489 


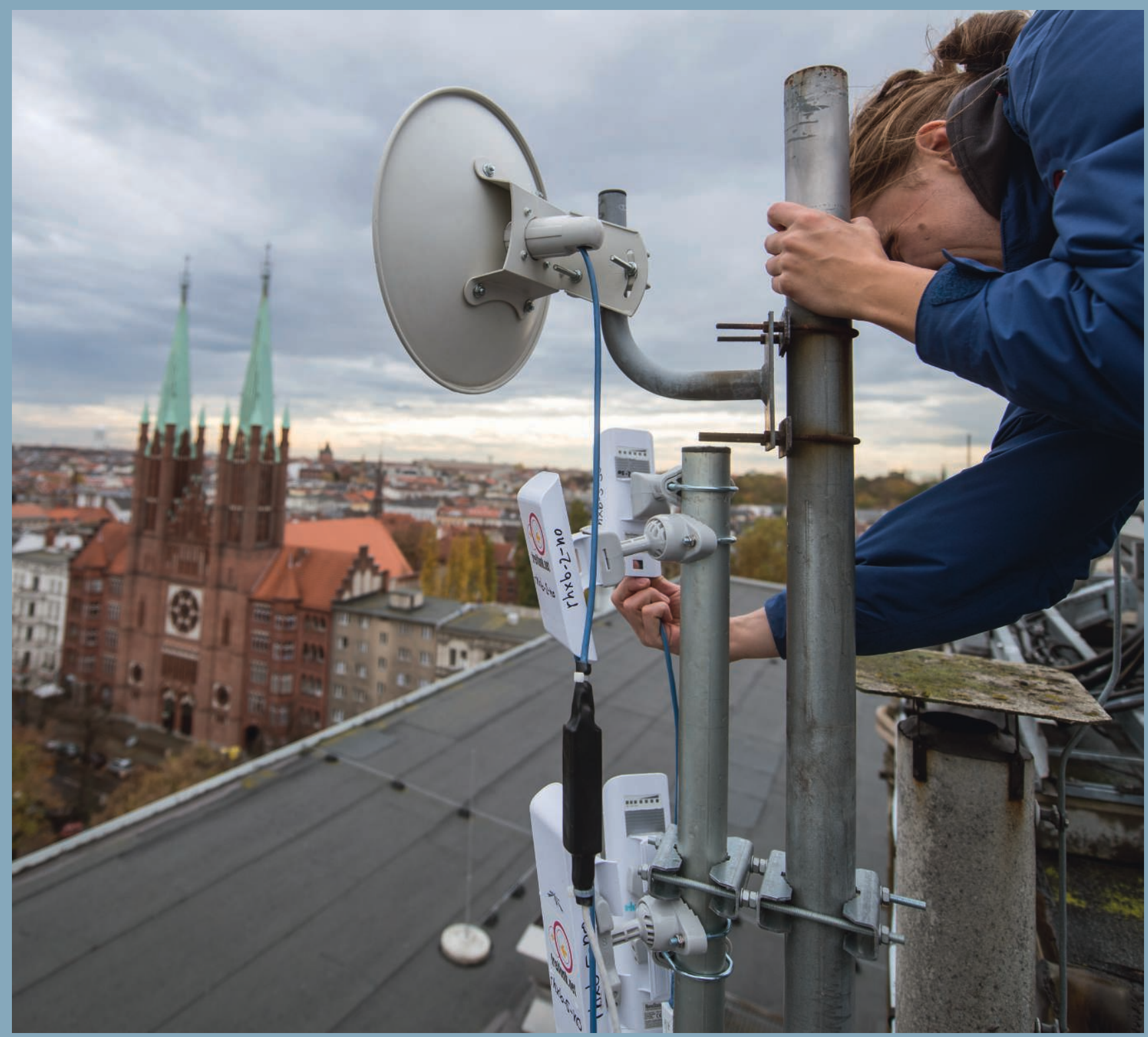




\section{Comment bâtir un internet low tech}

Plus de la moitié de la planète n'a pas accès au web, pourtant qualifié de « mondial » (« world wide web »). Pour l'heure, l'Internet est essentiellement un phénomène urbain, en particulier dans les pays « en développement». De fait, souvent, les entreprises de télécommunication hésitent souvent à étendre leur réseau à l'extérieur des villes pour des raisons multiples : coûts d'infrastructure élevés, faible densité de population, capacité à payer pour des services limitée, réseau électrique inexistant ou peu fiable. Même dans les pays « développés », la connectivité à l'Internet peut faire défaut dans certaines régions reculées.

Des sociétés de l'Internet font régulièrement la une en proposant de connecter ces régions isolées: Facebook avec des drones et Google au moyen de ballons à haute altitude. Outre les problèmes technologiques majeurs que posent ces projets, c'est leur caractère commercial qui suscite les plus vives objections car, si Google et Facebook souhaitent connecter davantage d'internautes, c'est à l'évidence pour accroître leurs recettes. Facebook en particulier est sous le feu des critiques, car son réseau encourage l'utilisation du site de l'entreprise et bloque la plupart des applications internet concurrentes ${ }^{1}$.

Parallèlement, plusieurs groupes de recherche ainsi que des amateurs passionnés par les réseaux ont conçu et mis en œuvre des technologies alternatives pour résoudre ces problèmes. Bien qu'on leur accorde beaucoup moins d'attention, ces réseaux low tech très économes en énergie ont prouvé leur utilité. Contrairement aux projets des sociétés de l'Internet, ils sont mis au point par de petites organisations ou par les internautes eux-mêmes, et ouverts à tous afin de profiter aux usagers et non à une poignée de grandes sociétés. 


\section{Réseaux wifi à grande distance}

La plupart des réseaux low tech reposent sur la technologie wifi, celle-là même qui permet l'accès mobile à internet dans la majorité des foyers occidentaux. La norme wifi a été pensée pour des communications de données à courte distance (environ $30 \mathrm{~m}$ ), mais sa portée peut être étendue en modifiant, dans le protocole, la couche de commande d'accès au support physique (MAC) et en utilisant des suralimenteurs-amplificateurs ainsi que des antennes directives (Hussain et al. 2015).

La plus longue liaison wifi non amplifiée est une connexion sans fil, point à point, de 384 km mise en place il y a quelques années au Venezuela, entre Pico El Águila et Platillón (Pietrosemoli 2011) ${ }^{2}$. Cependant, les réseaux à grande distance reposant sur la technologie wifi consistent le plus souvent en une combinaison de liaisons point à point beaucoup plus courtes, n'excédant pas $100 \mathrm{~km}$. Ces liaisons sont combinées pour créer de grands réseaux à plusieurs bonds. Les liaisons point à point, qui constituent la dorsale du réseau wifi à longue portée, sont associées à des antennes équidirectives qui distribuent individuellement le signal aux différents foyers ou organismes publics des communautés.

Avec les liaisons wifi à grande distance, il est nécessaire d'être en visibilité directe pour établir une connexion. Vue sous cet angle, cette technologie ressemble au télégraphe optique du xviII siècle $^{3}$. Lorsque deux points ne sont pas en visibilité directe, il est nécessaire d'installer entre eux un relais intermédiaire. Si le relief et des obstacles l'exigent, des stations pivots supplémentaires sont éventuellement installées ${ }^{4}$.

En règle générale, les liaisons point à point sont réalisées au moyen de deux antennes directives, l'une pointant vers le nœud suivant du réseau et l'autre vers le nœud précédent. Les nœuds peuvent être dotés de plusieurs antennes, une par liaison point à point fixe orientée vers chacun des nœuds voisins (Patra et al. 2007). Cette configuration permet l'utilisation de protocoles de routage maillé, qui ont la faculté de sélectionner de façon dynamique, parmi les liaisons libres, celle qu'il convient d'emprunter (Vega et al. 2002).

Le plus souvent, les nœuds de répartition consistent en une antenne sectorielle (version réduite des dispositifs que l'on peut voir sur les pylônes de téléphonie mobile) ou en un routeur wifi classique, auquel s'ajoute un certain nombre de récepteurs installés dans la communauté à desservir ( $c f$. note 4). Pour les communications wifi à courte distance, il n'est pas nécessaire que l'émetteur et le récepteur soient en visibilité directe 5 .

Pour offrir aux usagers un accès à l'Internet mondial, il convient de connecter un réseau wifi à grande distance à la dorsale principale de l'Internet en utilisant au minimum une liaison de raccordement ou un nœud passerelle, par exemple une connexion téléphonique ou à large bande passante (ADSL, fibre ou satellite). Lorsque l'établissement de cette liaison est impossible, les usagers ne peuvent pas accéder au réseau internet, mais ils ont toujours la possibilité de communiquer entre eux et de consulter des sites web hébergés sur des serveurs locaux ${ }^{6}$. 


\section{Avantages du wifi à grande distance}

Aujourd'hui généralisée, la norme wifi se caractérise par des volumes de production gigantesques. Le wifi à grande distance offre un débit élevé (jusqu'à $54 \mathrm{Mb} / \mathrm{s}$ ) conjugué à des coûts d'investissement très faibles. On peut construire une installation wifi en utilisant des antennes et des cartes wifi sur étagère pour un prix très modique (Brewer et al. 2005). Une autre solution consiste à assembler des composants issus de matériels mis au rebut (routeurs, paraboles satellites ou ordinateurs portables d'ancienne génération). Les protocoles tels que WiLDNet s'exécutent sur un processeur cadencé à $266 \mathrm{MHz}$ avec 128 Mo de mémoire seulement ; un ancien ordinateur fait donc l'affaire (Patra et al. op. cit.).

Comme les nœuds wifi sont légers, il n'est pas nécessaire de les installer sur des tours onéreuses, ce qui permet de réduire les coûts d'investissement et de limiter l'impact des infrastructures à bâtir (ibid.). On trouve depuis peu des unités tout-en-un combinant une antenne, une carte wifi et un processeur, qui facilitent grandement l'installation. Pour construire un relais, il suffit de connecter plusieurs unités de ce type au moyen de câbles Ethernet, qui servent à acheminer aussi bien le signal que l'alimentation (cf. note 4). Les unités offrent peu de prise au vent et peuvent donc être installées dans des tours ou sur des pylônes de faible diamètre (Pietrosemoli op. cit.).

De surcroît, le wifi à grande distance étant peu gourmand en énergie, les coûts d'exploitation sont peu élevés. Classiquement, une installation de type pylône composée de deux liaisons à grande distance et d'une ou deux cartes wifi pour la diffusion locale consomme environ 30 watts (cf. note 4 ; Surana et al. 2008). C'est pourquoi dans certains réseaux low tech, les nœuds sont entièrement alimentés par des panneaux solaires et des batteries. Un autre avantage du wifi à grande distance est l'utilisation de fréquences non assujetties à licence (2,4 et $5 \mathrm{GHz}$ ), ce qui permet d'éviter les négociations avec les opérateurs de télécommunication et avec les États. Cette simplicité, combinée au faible coût, permet à quiconque, ou presque, de construire et d'exploiter ce type de réseau (cf. note 5).

\section{Les réseaux wifi à grande distance dans les pays pauvres}

Les premiers réseaux wifi à grande distance ont été créés il y a entre dix et quinze ans. Dans les pays pauvres, ils se déclinent en deux grandes catégories. La première regroupe les réseaux destinés à offrir un accès internet aux populations de villages isolés. C'est le cas, par exemple, de l'un des plus grands réseaux wifi au monde, le réseau Akshaya, en Inde, qui dessert la totalité de l'État du Kérala. L'infrastructure repose sur quelque 2500 « centres d'accès informatique » ouverts à la population locale (dans cette région, le pourcentage des habitants possédant un ordinateur est très faible) ${ }^{7}$. 

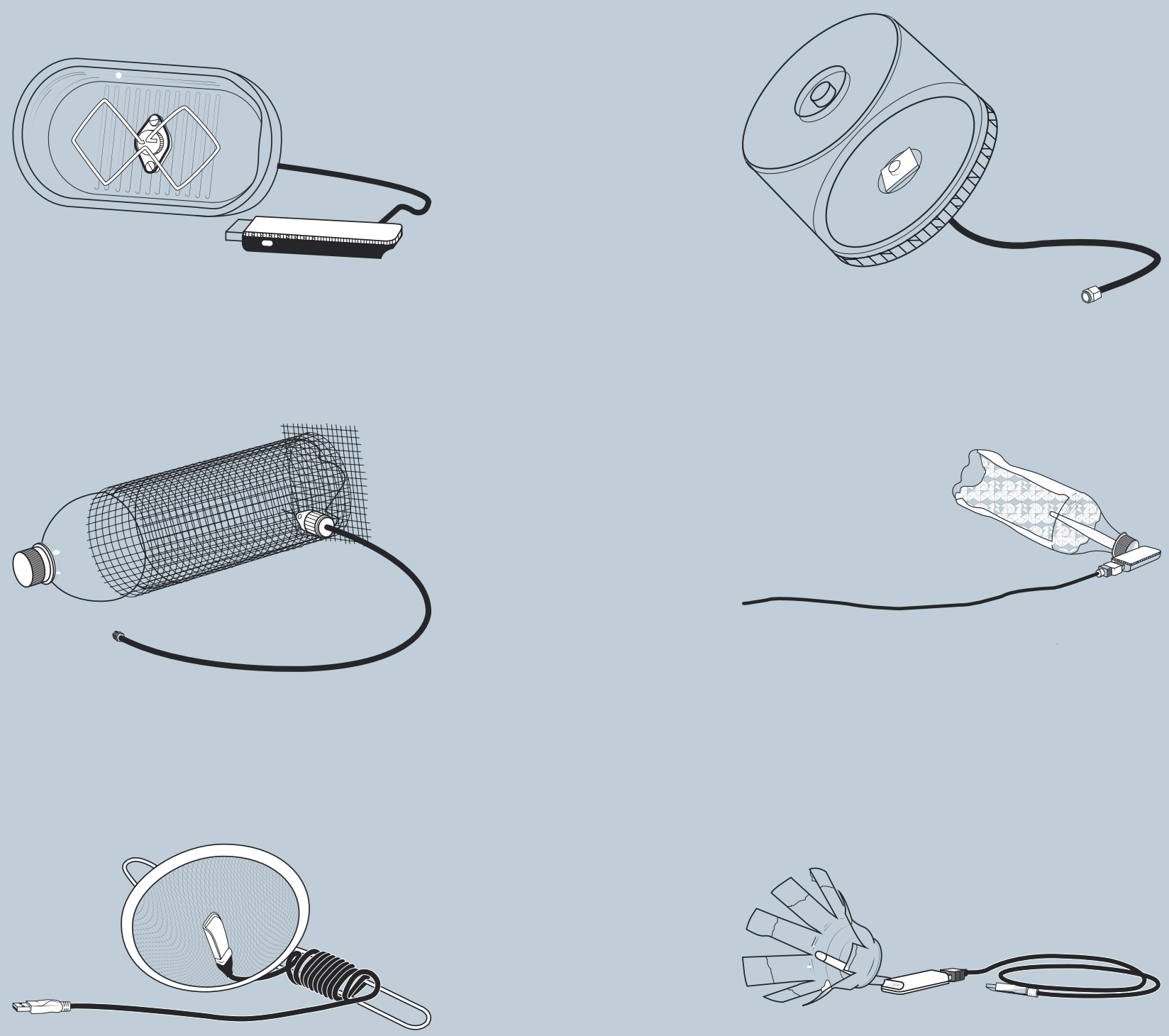

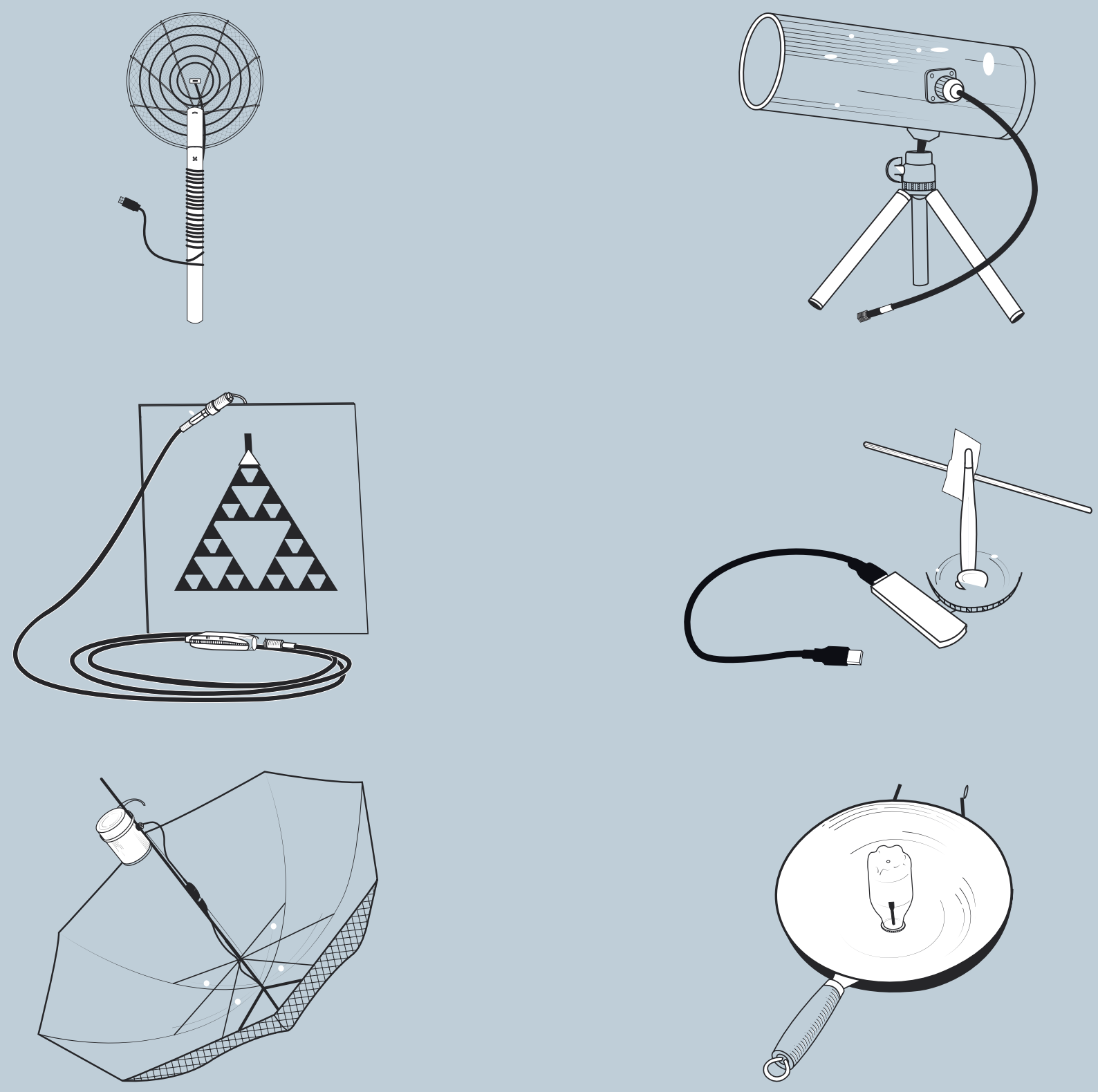
En Inde également, les réseaux Airjaldi offrent un accès internet à environ 20000 usagers de six États, vivant tous dans des régions isolées ou à la topographie difficile. La distance qui sépare les nouds de ce réseau, pour la plupart alimentés par l'énergie solaire, peut atteindre $50 \mathrm{~km}$, voire plus ${ }^{8}$. Dans certains pays africains, des réseaux wifi locaux distribuent l'accès internet à partir de passerelles satellitaires (Anand et al. 2012, Ndlovu et al. 2009).

La seconde catégorie des réseaux wifi à grande distance installés dans les pays pauvres apporte la télémédecine aux communautés isolées. De fait, dans les régions reculées, les soins sont souvent prodigués dans des postes médicaux mal équipés, par des techniciens de santé à peine formés (Rey-Moreno et al. 2011). Les réseaux wifi à grande distance permettent de relier des hôpitaux de ville à ces postes de soins périphériques. Les médecins peuvent donc assister les techniciens de santé grâce au transfert de fichiers à haute résolution et à des outils de communication en temps réel reposant sur l'audio ou la vidéo.

Dans cette catégorie, on peut mentionner la liaison, créée en 2007, entre Cabo Pantoja et Iquitos dans la province du Loreto au Pérou. Ce réseau de $450 \mathrm{~km}$ est composé de dix-sept tours séparées de 16 à $50 \mathrm{~km}$. La ligne, qui relie quinze avant-postes médicaux de villages isolés avec l'hôpital central d'Iquitos, est destinée au diagnostic à distance (Rey-Moreno et al. op. cit., Prieto-Egido et al. 2014). Tous les équipements sont alimentés par des panneaux solaires (ibid., Simó-Reigadas et al. 2008). D’autres réseaux de télémédecine wifi à grande distance ont été mis en place avec succès en Inde, au Malawi et au Ghana (Zennaro et al. 2008, Voyiatzis 2012).

\section{Les réseaux wifi communautaires en Furope}

Dans les pays pauvres, les réseaux low tech sont l'œuvre d'ONG, des pouvoirs publics, d'universités ou d'entreprises. Dans les régions isolées des pays riches, en revanche, la plupart des réseaux wifi à longue distance sont des réseaux dits « communautaires », à savoir des réseaux dont l'infrastuctrue est bâtie, détenue, alimentée et entretenue par les usagers eux-mêmes. Ces réseaux reposent sur le partage réciproque des ressources : les participants peuvent construire leur propre noud et le connecter gratuitement au réseau, à condition que ce dernier autorise aussi le trafic des autres membres. Chaque nœud fait office de routeur wifi et offre à tous les usagers ainsi qu'aux nœuds auxquels il est connecté des services de réacheminement IP et une liaison de données (Vega et al. op. cit., Jimenez et al. 2013).

Par conséquent, à chaque nouvel utilisateur, le réseau s'agrandit sans planification d'ensemble a priori. Les réseaux communautaires se développent de la base vers le sommet en fonction des besoins de leurs utilisateurs et des nouvelles exigences structurelles, à mesure que des nouds et des liaisons sont ajoutés ou perfectionnés. Le problème se résume à connecter le nœud d'un nouveau participant à celui d'un usager existant. Lorsqu'un nœud est mis sous tension, il découvre ses voisins, s'attribue une adresse IP unique et calcule, en tenant compte de la qualité des liaisons, les meilleurs itinéraires à emprunter pour atteindre le reste du réseau. Les 
réseaux communautaires sont accessibles à tous, moyennant parfois un accord ouvert d'échange de trafic (Vega et al. op. cit., cf. note 5, Simó-Reigadas et al. op. cit., Jimenez et al. op. cit.).

Il semble, malgré l'absence de statistiques fiables, que les réseaux communautaires connaissent un certain succès. L'Europe en compte plusieurs de grande taille, notamment Guifinet (Espagne), Athens Wireless Metropolitan Network (Grèce), FunkFeuer (Autriche) et Freifunk (Allemagne) (Vega et al. op. cit., Jimenez et al. op. cit., Braem et al. 2013) ${ }^{9}$. Le premier est le plus vaste réseau wifi à grande distance au monde, avec plus de $50000 \mathrm{~km}$ de liaisons, même si une petite partie est réalisée à base de fibre optique. La quasi-totalité de ce réseau est située dans les Pyrénées catalanes, l'une des régions les moins peuplées d'Espagne. Créé en 2004, il compte aujourd'hui près de 30000 nœuds, contre 17000 en 2012 (Vega et al. op. cit., Jimenez et al. op. cit.).

Guifi.net offre un accès internet aux particuliers, aux entreprises, aux administrations et aux universités. En principe, ce sont les utilisateurs qui s'occupent de l'installation, de l'alimentation et de l'entretien ; en pratique, ils sont assistés par des équipes de bénévoles et, parfois, par des installateurs professionnels. Certains nœuds ainsi que des mises à jour du réseau dorsal ont été réalisés grâce au financement participatif, apporté en l'occurrence par des bénéficiaires indirects du réseau (Vega et al. op. cit., Jimenez et al. op. cit.).

\section{Performances des réseaux low tech}

Quelles sont les performances des réseaux low tech et quels services offrent-ils? La bande passante disponible par utilisateur peut varier dans des proportions considérables, selon, entre autres, la largeur de bande du ou des nœuds passerelles et le nombre d'usagers. Les réseaux wifi à grande distance destinés à la télémédecine dans les pays pauvres comptent peu d'utilisateurs et possèdent une liaison de raccordement performante. Ils offrent donc un débit élevé (+ $40 \mathrm{Mb} / \mathrm{s})$ et des performances analogues aux connexions par fibre que l'on trouve dans les pays développés. Une étude portant sur une petite partie du réseau communautaire Guifi.net, qui compte des dizaines de nœuds passerelles et des milliers d'utilisateurs, indique un débit moyen de $2 \mathrm{Mb} / \mathrm{s}$, ce qui est comparable à une connexion ADSL relativement lente. Le débit réel par usager est compris entre $700 \mathrm{~kb} / \mathrm{s}$ et $8 \mathrm{Mb} / \mathrm{s}$ (Pitarch 2013).

Cela étant, certains réseaux low tech qui fournissent un accès internet à une large base d'utilisateurs dans des pays en développement ont une bande passante par usager beaucoup plus limitée. Par exemple, un campus universitaire du Kérala (Inde) est doté d'une connexion internet à $750 \mathrm{~kb} / \mathrm{s}$ que se partagent 3000 professeurs et étudiants connectés sur 400 ordinateurs, sachant qu'aux heures de pointe la quasi-totalité des machines est utilisée.

Par conséquent, dans le cas le plus défavorable, le débit moyen disponible par machine, est de 1,9 kb/s environ, débit que l'on peut qualifier de lent, même en comparaison d'une connexion par le réseau commuté $(56 \mathrm{~kb} / \mathrm{s})$. Et encore ce cas correspond-il à une connectivité de très bonne 
qualité par rapport à ce qui existe en général en milieu rural dans les pays pauvres (Chen et al. 2009). Qui plus est, ce type de réseau est souvent confronté à des interruptions intermittentes du réseau électrique.

Dans ces circonstances, même les applications internet les plus courantes affichent de mauvaises performances, et certaines ne fonctionnent pas du tout. Le modèle de communication de l'Internet repose sur un ensemble de postulats relatifs au réseau appelé « suite de protocoles TCP/IP ». Parmi ces postulats figurent notamment l'existence d'un trajet bidirectionnel de bout en bout entre la source (par exemple, un serveur web) et la destination (l'ordinateur de l'utilisateur), des temps de propagation aller-retour courts et des taux d'erreur faibles.

Or, dans les pays pauvres, de nombreux réseaux low tech ne respectent pas ces postulats. Ils se caractérisent par une connectivité intermittente ou par un « partitionnement du réseau » (absence d'un trajet de bout en bout entre la source et la destination), des temps de propagation longs et variables et des taux d'erreur élevés (Voyiatzis op. cit., Fall 2003, Warthman (Voyiatzis op. cit., Fall 2003, Warthman 2012).

\section{Réseaux tolérants aux retards de propagation}

Même dans ces conditions, il est possible de fournir un accès à l'Internet. Les difficultés techniques peuvent être résolues en abandonnant le modèle de connexion permanente des réseaux traditionnels au profit de systèmes fondés sur la communication asynchrone et la connectivité intermittente. Les "réseaux tolérants aux retards de propagation », ou DTN, n'utilisent pas TCP, mais des protocoles spécialisés, qui s'exécutent au-dessus des protocoles de bas niveau. Les problèmes de connectivité intermittente et de retards importants sont résolus au moyen de la commutation de messages avec enregistrement et retransmission.

Les données sont retransmises d'un nœud à l'autre en passant, sur chaque nœud, par une zone de stockage, le long d'un trajet qui mène à la destination. Contrairement aux routeurs internet traditionnels, qui mémorisent les paquets entrants pendant quelques millisecondes sur des puces mémoire, les nœuds des réseaux tolérants aux retards sont dotés d'un stockage permanent (disques durs par exemple) où les données peuvent être conservées indéfiniment (Fall op. cit., Warthman op. cit.).

Les réseaux tolérants aux retards n'imposent pas qu'il existe un trajet de bout en bout entre la source et la destination : les données sont tout simplement transmises d'un nœud à l'autre. Si le nœud suivant est indisponible en raison d'importants retards de propagation ou d'une coupure d'alimentation, les informations sont stockées sur le disque dur du nœud courant jusqu'à ce que le nœud suivant redevienne disponible. Même si le temps total d'acheminement peut être long, les réseaux tolérants aux retards apportent la garantie que les données finiront par atteindre leur destination. 
De plus, les dépenses d'investissement et la consommation d'énergie étant moindres, l'utilisation des ressources - qui sont limitées - est optimale. Les réseaux tolérants aux retards fonctionnent même lorsque la source d'énergie est intermittente et sont bien adaptés aux énergies renouvelables. Les nœuds du réseau peuvent donc, par temps clair ou venteux, être alimentés par des panneaux solaires ou des éoliennes, rendant inutile la mise en place d'accumulateurs d'énergie.

\section{Mules de données}

Les réseaux tolérants aux retards de propagation peuvent prendre des formes surprenantes, notamment s'ils s'appuient sur des moyens de communication inhabituels comme les « mules de données (Brewer et al. op. cit., Coutinho et al. 2014). Dans ce type de configuration, les messages sont acheminés d'un lieu à un autre en utilisant des moyens de transport conventionnels (bus, automobiles, motos, trains, bateaux, avions) et suivant le principe de l'enregistrement-retransmission.

Les réseaux DakNet et KioskNet, par exemple, utilisent des autocars comme mules de données (Chyau \& Raymond 2005, Seth et al. 2006). Dans de nombreuses régions en développement, les bus ruraux se rendent régulièrement dans des villes et des villages qui ne sont pas connectés au réseau. Pour remplacer une liaison internet hertzienne par une infrastructure de transport locale, il suffit d'équiper tous les véhicules d'un ordinateur, d'un dispositif de stockage et d'un nœud wifi mobile, et d'installer un nœud wifi fixe dans chaque village (Brewer et al. op. cit.).

Les données sortantes (e-mails envoyés, demandes de consultation de pages web, etc.) sont stockées dans un ordinateur local en attendant que le bus se trouve à portée du village. À ce moment, le nœud wifi fixe de l'ordinateur local envoie automatiquement les données au nœud wifi mobile installé à bord du bus. Plus tard, lorsque le bus arrive au niveau d'un concentrateur connecté à l'Internet, les données sortantes sont émises du nœud wifi mobile vers le nœud passerelle, puis vers l'Internet. Les données destinées au village suivent l'itinéraire inverse. Le conducteur du bus, qui, en somme, est un « conducteur de données », n’a pas besoin de compétences particulières. Les transferts de données s'effectuent de façon totalement transparente et sa seule tâche consiste à entrer dans la zone de couverture des nœuds (Chyau \& Raymond op. cit., Hasson et al. 2003).

Par rapport aux réseaux « perfectionnés » tolérants aux retards de propagation, les mules de données présentent plusieurs avantages. Ces réseaux wifi « itinérants » reposent sur des dispositifs de radiocommunication à bas prix, de petite taille et de faible puissance, qui, puisqu'ils fonctionnent sans visibilité directe, ne requièrent pas l'installation de tours. Ils sont donc plus économes que les autres réseaux low tech, tant sur le plan des investissements que sur celui de la consommation en énergie (Chyau \& Raymond op. cit., Hasson et al. op. cit., Bhole \& Wankhade 2015). 


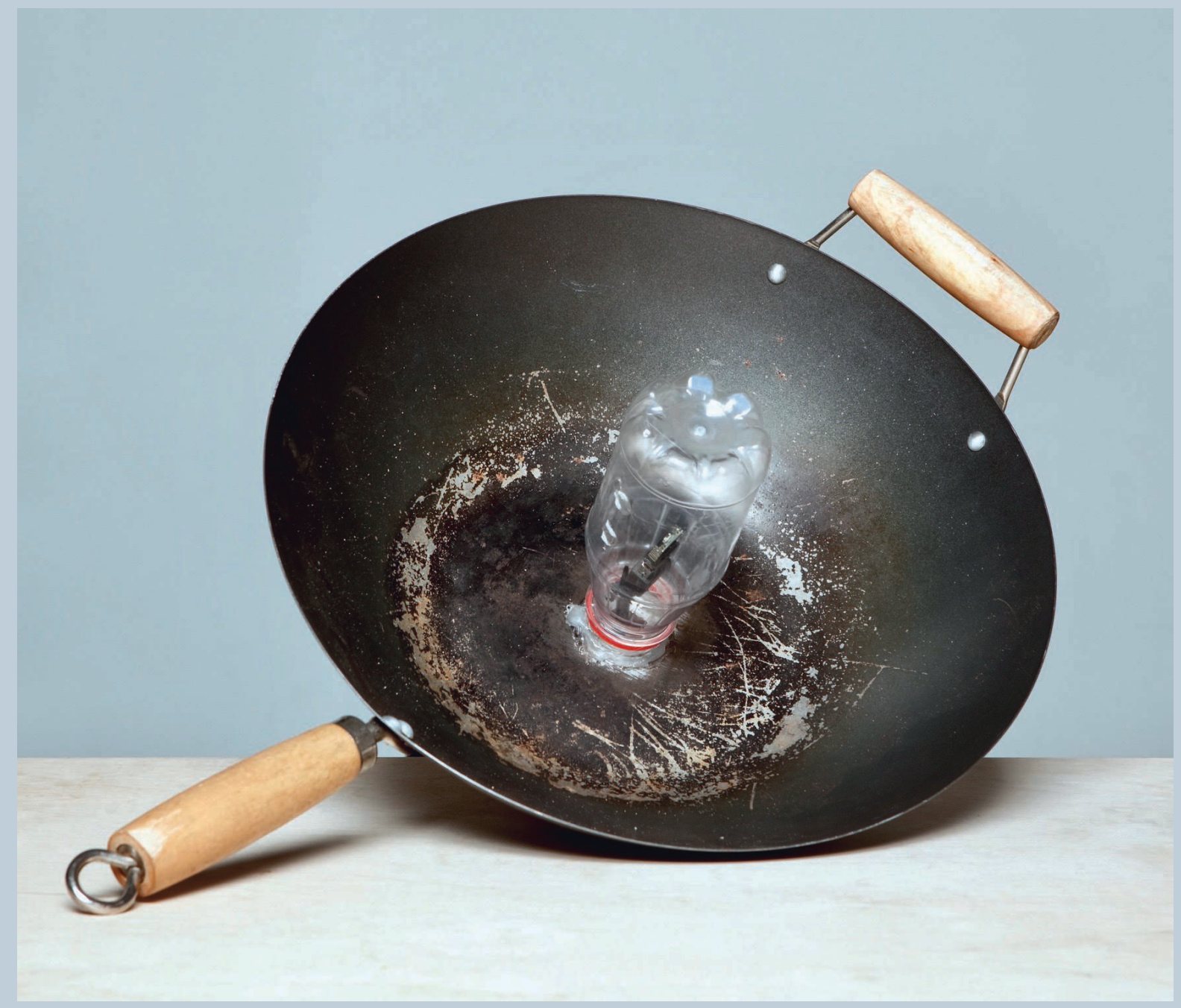



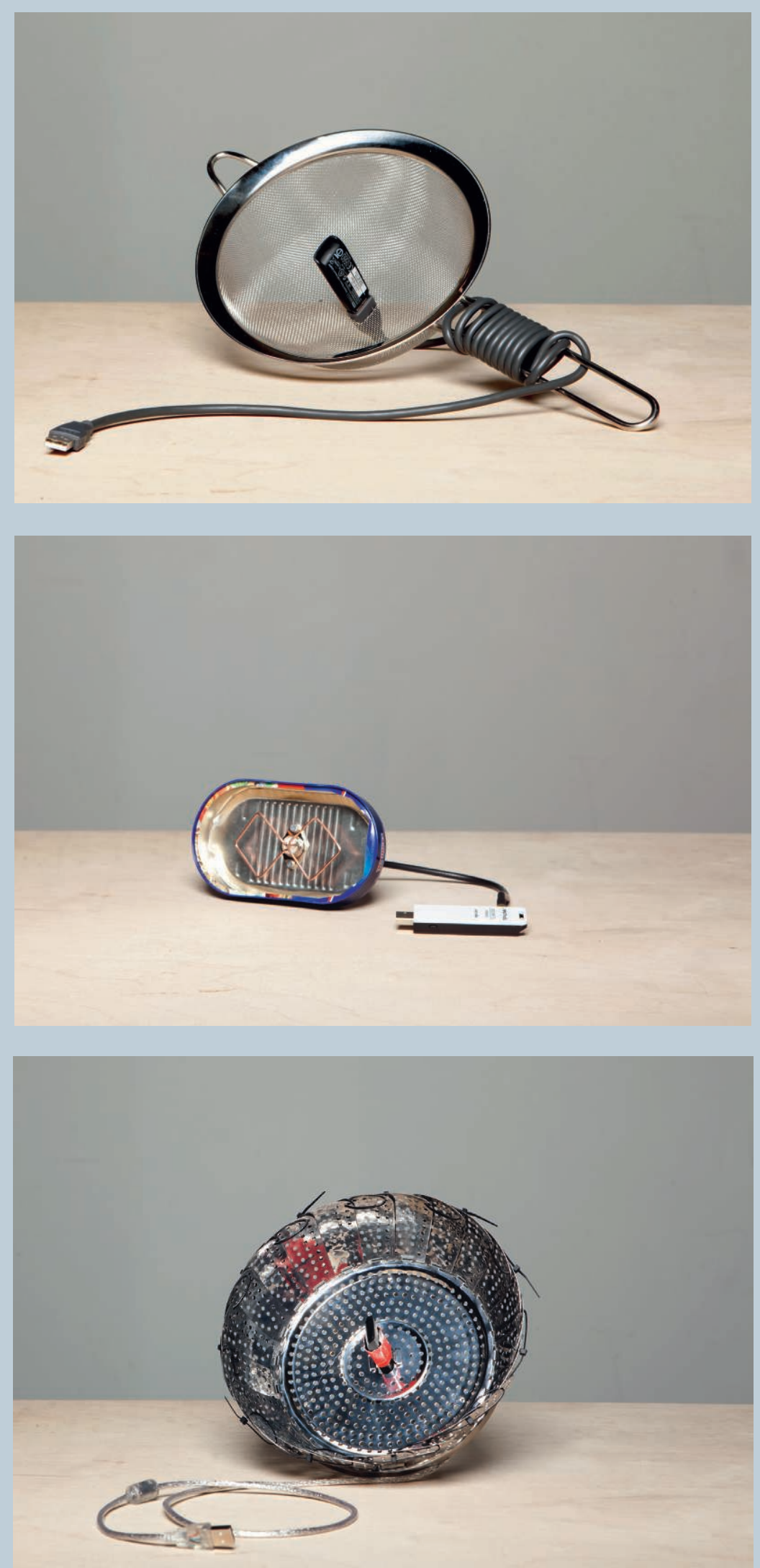
De plus, les liaisons wifi à courte distance offrent une bande passante plus large que les liaisons wifi à longue distance ; les mules de données sont donc plus adaptées aux transferts de fichiers volumineux. En moyenne, lorsqu'un bus passe à proximité d'un nœud wifi fixe, 20 Mo de données peuvent être transférés dans les deux sens (Chyau \& Raymond op. cit., Wankhade op. cit.). En revanche, la latence (temps d'attente entre l'envoi et la réception de données) est généralement supérieure à celle des liaisons wifi à longue distance. Si un seul bus passe une fois par jour à proximité du village à desservir, le temps de latence est de 24 heures.

\section{Logiciels tolérants aux retards de propagation}

Un réseau tolérant aux retards de propagation (DTN), quelle que soit sa forme, nécessite un nouveau logiciel, c'est-à-dire un ensemble d'applications pouvant s'exécuter sans cheminer de manière continue par un réseau connecté de bout en bout (Brewer et al. op. cit.). Ces applications spéciales sont aussi utilisées dans les réseaux synchrones à bande étroite. Asynchrone par nature, la messagerie électronique est relativement facile à adapter à une connectivité de type intermittent. La solution consiste à mémoriser les messages sortants au niveau du client e-mail compatible DTN jusqu'à ce qu'une connexion soit disponible. Même si les courriels mettent plus de temps à atteindre leur destination, l'expérience de l'utilisateur reste pour l'essentiel inchangée.

La navigation et la recherche sur le web nécessitent des adaptations supplémentaires. Par exemple, la plupart des moteurs de recherche s'attachent à optimiser la vitesse, en partant du principe qu'en règle générale, les utilisateurs examinent rapidement les liens affichés, puis lancent immédiatement une deuxième recherche si le résultat de la première n'est pas satisfaisant. Cela étant, les recherches interactives multiples seraient difficiles à réaliser avec des réseaux intermittents (Chen et al. op. cit., Thies et al. 2002). Les moteurs de recherche asynchrones s'attachent donc à optimiser la bande passante plutôt que le temps de réponse (Chen et al. op. cit., Chyau $\&$ Raymond op. cit., Hasson et al. op. cit., Thies et al. op. cit., Teevan et al. 2013). Par exemple, le système RuralCafe désynchronise le processus en effectuant de multiples tâches de recherche hors ligne et en affinant la requête à partir d'une base de données de recherches similaires. Il ne récupère des informations du réseau que lorsque cela est absolument nécessaire.

Certains navigateurs compatibles DTN téléchargent non seulement la page web explicitement demandée par l'usager, mais aussi les pages vers lesquelles pointent les liens qu'elle contient (Chyau \& Raymond op. cit.). D'autres navigateurs sont optimisés pour renvoyer des résultats occupant une faible largeur de bande ; dans ce cas, les résultats sont produits par filtrage, analyse et compression au niveau du serveur. Un effet analogue peut être obtenu en utilisant un service tel que Loband, qui se charge de supprimer toutes les images, vidéos, publicités et autres boutons vers des réseaux sociaux figurant dans les pages web pour ne laisser que le contenu textuel (Chen et al. op. cit.). 
La navigation et la recherche sur les réseaux intermittents peuvent aussi être améliorées par des mécanismes de cache local (mémorisation de pages déjà téléchargées) et de pré-extraction (téléchargement de pages qui pourraient être demandées ultérieurement) (Chen et al. op. cit.). De nombreuses autres applications internet pourraient être adaptées aux réseaux intermittents: remplissage de formulaires électroniques, échanges avec les sites de commerce en ligne, logiciels de blog, téléchargements de fichiers volumineux, médias sociaux, etc. (Brewer et al. op. cit., Chyau \& Raymond op. cit.). Toutes ces applications pourraient rester opérationnelles, au détriment toutefois de la vitesse.

\section{Sneakernets}

À l'évidence, il est impossible d'adapter les applications temps réel - comme la téléphonie sur internet, la transmission de médias en continu, le chat ou la vidéoconférence - aux réseaux intermittents qui sont limités à une communication de type asynchrone. De même, il est difficile d'exécuter ce type d'applications sur des réseaux synchrones à bande étroite. Or ce sont précisément ces applications qui, pour l'essentiel, font augmenter la consommation d'énergie de l'Internet. On pourrait donc dire que cette incompatibilité avec les réseaux low tech est une bonne chose.

Bon nombre de ces applications pourraient être restructurées. Si la prise en charge de conversations audio ou vidéo en temps réel n'est pas envisageable, il est en revanche parfaitement possible d'envoyer et de recevoir des messages audio ou vidéo. De même, si ces réseaux ne sauraient diffuser des médias en continu, le téléchargement d'albums et de clips de musique reste possible. De plus, ces fichiers pourraient être «transmis » au moyen de la technologie internet la moins sophistiquée qui soit, à savoir le «sneakernet ». Dans un sneakernet, les données numériques sont transmises « sans fil » à l'aide d'un support de stockage : disque dur, clé USB, carte flash, CD ou DVD. Avant l'arrivée de l'Internet, tous les fichiers informatiques étaient échangés via un sneakernet, au moyen d'une bande magnétique ou d'un disque souple.

À l'instar des réseaux de type mules de données, les sneakernets nécessitent un véhicule, un messager non motorisé ou un animal (pigeon voyageur, etc.). Mais dans un sneakernet, il n'y a pas de transfert automatique de données entre le nœud mobile (par exemple, un véhicule) et les nœuds fixes (expéditeur et destinataire). Les données doivent d'abord être transférées de l'ordinateur de l'expéditeur vers un support de stockage portable, puis, à leur arrivée, du support de stockage vers l'ordinateur du destinataire (Chyau \& Raymond op. cit.). Par conséquent, les sneakernets nécessitent une intervention manuelle et se prêtent donc mal à de nombreuses applications internet.

Il y a cependant des exceptions. Par exemple, il est possible de visionner un film sans le transférer vers le disque dur de l'ordinateur; il suffit pour cela de le lire directement à partir du disque dur portable ou d'insérer un disque dans le lecteur de DVD. De plus, le sneakernet offre un autre avantage de poids : de tous les réseaux low tech, il est celui qui possède la bande 


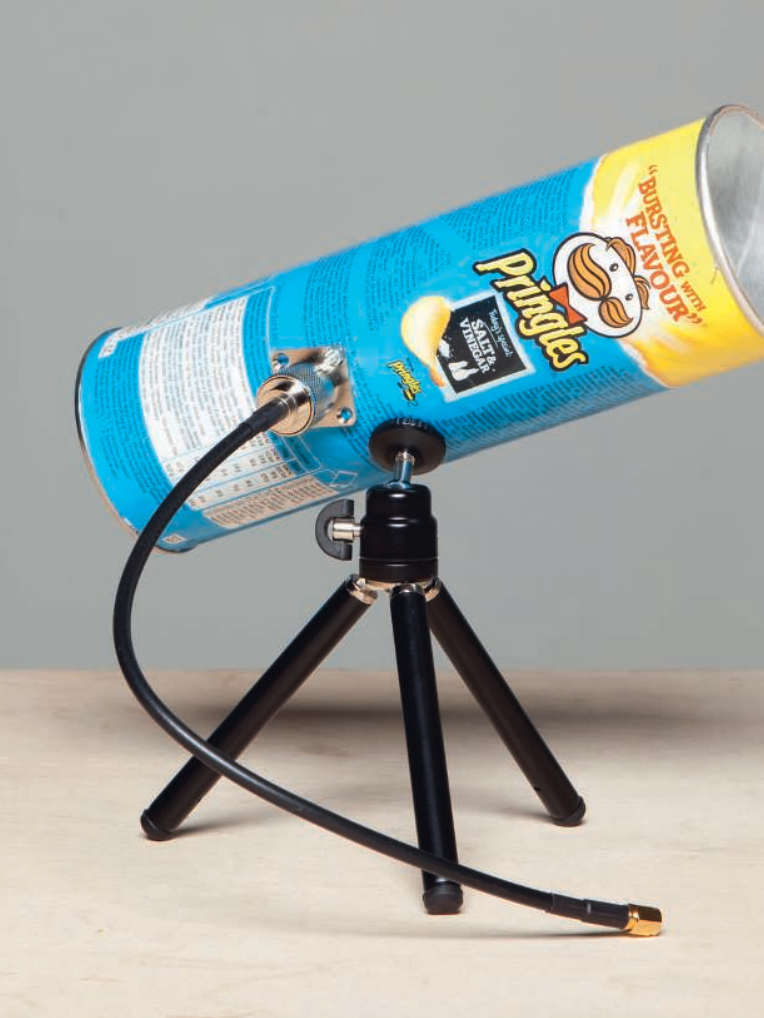



passante la plus large. Il est de ce fait parfaitement adapté à la diffusion de films, de jeux pour ordinateur et autres fichiers volumineux. En réalité, lorsque de très gros fichiers sont échangés, les sneakernets sont même plus performants que la plus rapide des connexions internet par fibre optique. Et pour des débits internet plus faibles, ils restent intéressants pour la transmission de fichiers beaucoup plus petits.

Les progrès technologiques ne remettront pas en cause les atouts des sneakernets. En effet, les supports de stockage numériques évoluent au moins aussi vite que les connexions internet et ces deux canaux améliorent la communication de façon égale.

\section{Réseaux résilients}

Étant donné que la plupart des réseaux low tech sont destinés aux régions où la seule autre solution est de renoncer purement et simplement à toute connexion internet, leur utilité ne peut être passée sous silence. L'Internet tel que nous le connaissons dans les pays industrialisés est le produit d'une offre énergétique abondante, d'une infrastructure électrique robuste et d'une croissance économique soutenue. L'Internet high tech offre certes des services sophistiqués par rapport aux réseaux low tech, mais il ne pourra pas survivre si ces conditions restent inchangées. Il est de ce fait extrêmement vulnérable.

Lorsque l'approvisionnement en combustibles fossiles est interrompu, que l'infrastructure électrique se dégrade, que l'économie se grippe ou que d'autres catastrophes surviennent, les réseaux low tech peuvent, selon leur degré de résilience, rester opérationnels. Cet internet rudimentaire permet, entre autres, de naviguer sur le web, d'envoyer et de recevoir des courriels, de faire des achats en ligne et de diffuser du contenu. Parallèlement, les mules de données et les sneakernets peuvent être utilisés pour la diffusion de fichiers volumineux (vidéos, etc.). Du reste, un vaisseau cargo ou un train remplis à ras bord de supports de stockage informatiques surpasseraient tout réseau numérique en termes de débit, de coûts et d'efficience énergétique. Et s'il arrivait que ces infrastructures de transport deviennent indisponibles, nous pourrions toujours compter sur les messagers à pied, les vélos cargos et les bateaux à voile.

Un tel système hybride constitué d'applications en ligne et hors-ligne resterait un réseau de communication très puissant, en rien comparable à ce que nous avons connu, même à la fin du xx ${ }^{e}$ siècle. Et même dans le pire des scénarios, à savoir une panne généralisée de l'infrastructure internet, les réseaux isolés low tech auraient encore une grande utilité dans les communications locales et régionales. De plus, ces réseaux peuvent récupérer des données provenant de réseaux distants, par échange de supports de stockage portables. En réalité, l'Internet peut être plus ou moins perfectionné selon les ressources qu'on lui consacre. 


\section{Traduction}

Techniques\& Culture remercie Kris de Decker dont l'aimable autorisation nous a permis d'éditer en français cet article initialement paru en langue anglaise dans Low-Tech Magazine le 26 octobre 2015, [en ligne: lowtechmagazine.com/2015/10/ how-to-build-a-low-tech-internet.html] ainsi qu'Olivier Morice pour la qualité de son travail de traduction.

\section{Notes}

1. Slashdot 2015 «Connecting the unwired world with balloons, satellites, lasers \& drones». En ligne: tech.slashdot.org/story/15/09/03/214256/ connecting-the-unwired-world-with-balloons-satellites-lasers-drones.

2. Cette liaison n'a pu être établie que grâce à l'altitude des deux points (4200 et $1500 \mathrm{~km}$ ) et à la planéité du terrain intermédiaire. La courbure de la Terre rend difficile la mise en place de liaisons point à point plus longues, car une visibilité directe entre les deux points est nécessaire.

3. Les ondes radioélectriques occupent, autour de la ligne optique, un volume qui doit être dépourvu d'obstacles. Ce volume est connu sous le nom d'ellipsoïde de Fresnel; sa taille grandit avec la distance entre les deux points terminaux et avec la longueur d'onde du signal, laquelle est inversement proportionnelle à la fréquence. Aussi est-il nécessaire de prévoir une «marge de manœuvre » supplémentaire pour la zone de Fresnel. Voir aussi la note 5.

4. Tegola Project 2007 A Brief Historiy of the Tegola Project. Scotland: HUBS. En ligne: www.tegola. org.uk/tegola-history.html.
5. GAIA - Global Access to the Internet for All, Internet draft, Internet Engineering Task Force (IETF), 2015. En ligne: trac.ietf.org/trac/irtf/wiki/gaia.

6. C'est ce qui est arrivé au réseau afghan JLINK lorsque les financements pour sa liaison satellitaire se sont taris en 2012. En ligne: www.wired. com/2012/05/jlink.

7. http://www.akshaya.kerala.gov.in/

8. http://main.airjaldi.com/

9. Il existe des réseaux plus petits en Écosse (Tegola), en Slovénie (wlan slovenija), en Belgique (Wireless Antwerpen) et aux Pays-Bas (Wireless Leiden), entre autres. On trouve en Australie le réseau Melbourne Wireless. En Amérique latine, il existe de nombreux exemples, comme le Bogota Mesh (Colombie) et le Monte Video Libre (Uruguay). Certains de ces réseaux sont interconnectés. C'est le cas des réseaux communautaires belge et néerlandais, et des réseaux slovène et autrichien. (Voir aussi Vega et al. 2002, Jimenez et al. 2013, Braem et al. 2013).

\section{I'auteur}

Kris De Decker est l'auteur de Low-tech Magazine, une publication en ligne qui refuse de supposer que chaque problème a une solution haute technologie. Il écrit aussi pour l'université de Lancaster (Royaume-Uni), qui mène des recherches sur les demandes d'énergie en lien avec les pratiques sociales, les infrastructures matérielles et les dispositions institutionnelles. Avant la création de Low-tech Magazine en 2007, Kris De Decker réalisait des reportages sur la science d'avant-garde et la technologie en tant que journaliste indépendant pour des journaux et des magazines. Il est né en Belgique et vit en Espagne.

\section{Iconographie}

Image d'ouverture. Un nœud wifi du réseau sans fil communautaire Freifunk, en cours d'installation à Berlin. (C) Wikipedia Commons.

Illustrations. CC by Lidia Pereira pour Pretty Fly For A Wi-fi. Images. CC by Roel Roscam Abbing pour
Pretty Fly for A Wi-fi. https://roelof.info/projects/(2014) Pretty_Fly_For_A_Wifi/.

Les deux séries de dessins et de photos illustrent un même objet: des antennes wifi artisanales, réalisées à partir de matériaux recyclés. 


\section{Références}

Anand, A., Pejovic, V., Belding, E. M. \& D. L. Johnson 2012 «VillageCell: Cost Effective Cellular Connectivity in Rural Areas», Proceedings of the Fifth International Conference on Information and Communication Technologies and Development (ICTD 2012) : 180-189. En ligne: DOI:10.1145/2160673.2160698.

Bhole, M. \&Wankhade, P. 2015 «DakNet. Architecture and Connectivity in Developping Nations » International Journal of Pure and Applied Research in Engineering and Technology (IJPRET) 3 (9) : 647-655. En ligne: ijpret. com/publishedarticle/2015/4/IJPRET - ECN 115.pdf.

Braem, B., Blondia, C., Bonicioli, J., Papathanasiou, S. et al. 2013 «A Case for Research with and on Community Networks» Computer Communication Review 43 (3): 68-73. En ligne: sigcomm.org/sites/default/files/ccr/ papers/2013/July/2500098-2500108.pdf.

Brewer, E., Demmer, M., Du, B., Ho, M. et al. 2005 «The case for technology in developing regions », Computer 38 (6) : 25-38. En ligne: cs.cmu.edu/\%7Emattkam/ lab/publications/Computer2005.pdf.

Chen, J., Subramanian, L., Li, J. 2009 « RuralCafe: Web Search in the Rural Developing World», Session: Query Processing - International World Wide Web Conference Committee (IW3C2), Madrid: 411-420. En ligne: ambuehler.ethz.ch/CDstore/www2009/proc/docs/ p4l1.pdf.

Chyau, C. \& Raymond, J.-F. 2005 «First Mile Solutions' Daknet Takes Rural Communities Online» What Works (Case Study) : 1-29. En ligne: share4dev.info/ telecentreskb/documents/4316.pdf.

Coutinho, M. M., Efrat, A., Johnson, T., Richa, A. \& L. Mengxue 2014 « Healthcare Supported by Data Mule Networks in Remote Communities of the Amazon Region » International Scholarly Research Notices: 1-8. DOI:10.1155/2014/730760.

Fall, K. 2003 «A Delay-Tolerant Network Architecture for Challenged Internets » in SIGCOMM'03 (August 25-29) Applications, Technologies, Architectures and Protocols for Computer Communications: 27-34. En ligne: kevinfall.com/seipage/papers/p27-fall.pdf.

Gao, L., Yu, S., Luan, T. H. \& Z. Wanlei 2015 Delay Tolerant Networks and Their Applications. SpringerBriefs in Computer Science. En ligne: citeulike.org/user/tnhh/ article/13517347. DOI:10.1007/978-3-319-18108-0.

Hasson, A., Fletcher, R. \& A. Pentland 2003 « DakNet. A Road to Universal Broadland Connectivity» : 1-9. En ligne: courses.media.mit.edu/2003fall/de/DakNetCase.pdf.

Hussain, I., Ahmed, Z.I., Saikia, D. K. et al. 2015 «A QoS-aware dynamic bandwidth allocation scheme for multi-hop WiFi-based long distance networks», Journal on Wireless Communications and Networking (Décembre): 160. DOI: 10.1186/s13638-015-0352-z.

Jiménez, J., Baig, R., Escrich, P., Khan, A. M. et al. 2013 «Supporting Cloud Deployment in the Guifi.net Community Network» : 1-3. En ligne: sics.se/\%7Eamir/files/download/papers/guifi.pdf.

Ndlovu, N., Thinyane, M. \& A. Terzoli 2009 «Deployment and Extension of a Converged WiMAX/WiFi Network for Dwesa Community Area South Africa» : 1-2. En ligne: citeseerx.ist.psu.edu/viewdoc/download?doi= 10.1.1.452.7357\&rep=repl\&type=pdf.

Patra, R., Nedevschi, S., Surana, S. et al. 2007 «WiLDNet: Design and Implementation of High Performance WiFi Based Long Distance Networks » : 1-14. 4th Symposium on Networked Systems Design and Implementation (NSDI 2007): Cambridge, MA. En ligne: tier.cs.berkeley. edu/docs/wireless/wild_multihop.pdf.

Pietrosemoli, E. 2011 «Long-Distance, Low-Cost Wireless Data Transmission », Radio Science Bulletin 339: 23-31. En ligne: ursi.org/files/RSBissues/RSB_339_2011_12. pdf.

Pitarch Miguel, P. 2013 Proxy performance analysis in a community wireless network. Master Thesis, UPC / EETAC Escola d'Enginyeria de Telecomunicació i Aeroespacial de Castelldefels / Universitat Politècnica de Catalunya. URI: hdl.handle.net/2099.1/19710.

Prieto-Egido, I., Simó-Reigadas, J., Liñán-Benítez, L., García-Giganto, V. \& A. Martínez-Fernández 2014 «Telemedicine Networks of EHAS Foundation in Latin America», Frontiers in Public Health 2 (188) : 1-9. DOI: org/10.3389/fpubh.2014.00188.

Rey-Moreno, C., Quispe-Tacas, R., Bebea-Gonzalez, I., Liñán-Benitez, L. et al. 2011 «A Telemedicine WiFi Network Optimized for Long Distances in the Amazonian Jungle of Peru », Extrem Com' 11 : 1-6. En ligne: www.ehas.org/wp-content/uploads/2012/01/ Extremecomm_sig_ISBN.pdf.

Seth, A., Kroeker, D., Zaharia, M., Guo, S. \& S. Keshav 2006 « Low-cost Communication for Rural Internet Kiosks Using Mechanical Backhaul» : MobiCom'06 The Twelfth Annual International Conference on Mobile 
Computing and Networking: 1-12. En ligne: people.csail. mit.edu/matei/papers/2006/mobicom_kiosks.pdf.

Simó-Reigadas, F. J., Martinez Fernandez, A., Osuna Garcia, P., Lafuente Sanz, S. et al. 2008 «The design of a wireless solar-powered router for rural environments isolated from health facilities » IEEE Wireless Communication 15 (3) : 24-30. DOI: 10.1109/ MWC.2008.4547519.

Surana, S., Patra, R. Nedevschi, S., Ramos, M. et al. 2008 «Beyond Pilots: Keeping Rural Wireless Networks Alive : 119-132. USENIX Association. En ligne: usenix.org/legacy/event/nsdi08/tech/full_papers/ surana/surana.pdf.

Teevan, J., Collins-Thompson, K., White, R. W., Dumais, S. T. \& Y. Kim, 2013 «Slow Search: Information Retrieval without Time Constraints» : 1-10. Proceedings of the Symposium on Human-Computer Interaction and Information Retrieval (HCIR'13). En ligne: cs.cmu.edu/\%7Eyubink/hcir2013.pdf. DOI:10.1145/2528394.2528395

Thies, W., Prevost, J., Mahtab, T., Cuevas, G. T. et al. 2002 «Searching the World Wide Web in Low-Connectivity
Communities» : 1-20. Cambridge: MIT. En ligne: tek. sourceforge.net/papers/tek-www02.pdf.

Vega, D., Cerdà-Alabern, L., Navarro, L. \& R. Meseguer 2002 « Topology Patterns of a Community Network: Guifi.net » : 1-8. En ligne: dsg.ac.upc.edu/sites/default/ files/1569633605.pdf.

Voyiatzis A. G. 2012 «A Survey of Delay -and DisruptionTolerant Networking Applications », Journal of Internet Engineering 5 (1): 331-344. En ligne: citeseerx.ist.psu. edu/viewdoc/summary?doi=10.1.1.463.6280.

Warthman, F. 2012 Delay -and Disruption- Tolerant Networks (DTNs). A Tutorial. Palo Alto: Warthman Associates / the Interplanetary Internet Special Interest Group (IPNSIG, ) : 3-35. En ligne : ipnsig.org/ wp-content/uploads/2012/07/DTN_Tutorial_v2.04. pdf.

Zennaro, M., Fonda, C., Pietrosemoli, E., Muyepa, A. et al. 2008 «On a long wireless link for rural telemedicine in Malawi », 6th International Conference on Open AccessLilongwe. OAI: Diva.org :kth-27171 : 1-5. En ligne: users.ictp.it/\%7Emzennaro/Malawi.pdf.

\section{Pour citer l'article}

De Decker, K. 2017 «Comment bâtir un internet low tech? », TechniquesE Culture 67 «ow tech? Wild tech! », p. 216-235. 